\section{EFECTOS DE LA GESTIÓN ORGANIZACIONAL \\ EN LA RENTABILIDAD \\ EN PYMES: EUIDENCIAS EMPRílCAS Y ALGUNAS CONSIDERACIONES TEÓRICAS}

FECHA DE RECEPCIÓN: 13 de mayo FECHA DE APROBACIÓN: 29 de julio Pp. 88-109
EFFECTS OF ORGANIZATIONAL MANAGEMENT ON PROFITABILITY IN PYMES; EMPIRIGAL EVIDENGES AND THEORETIGAL CONSIDERATIONS

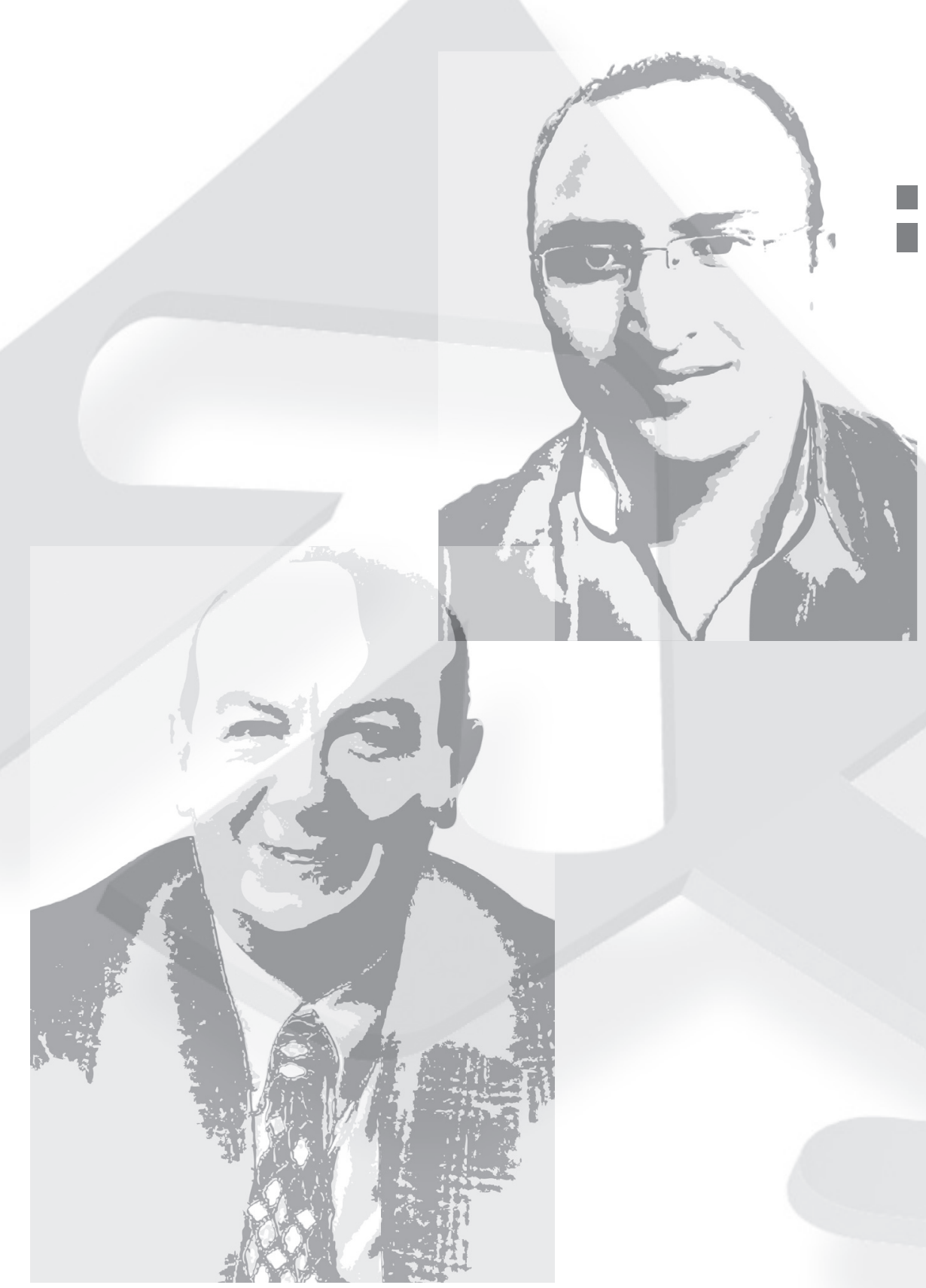

Willington Ortíz Rojas* Rafael I.Pérez Uribe**

* Magister en Economía Universidad de Los Andes Bogotá-Colombia; Economista con Énfasis en Finanzas Privadas,

Universidad EAN; Investigador Asociado y Docente Universidad EAN.

Publicaciones: Artículo en la revista Informe de Countura PyME: un buen panorama: lo que nos dicen los datos y la importancia de gestionar la información (2009); coautor del texto: Análisis de cadenas productivas, mercados y oportunidades de negocio (2007); coautor de la inversión extranjera directa en Colombia (2007).

**Ph.D. (c). Profesor asociado Universidad EAN. Director del grupo de investigación G3PYMES de la Universidad EAN. 


\section{RESUMEN}

ualquier resultado empresarial deviene de un proceso gerencial previo, el cual necesitó de una estructura organizacional que proporcionara forma y acción a los procesos de comunicación y decisión, acordados a su vez al interior de la figura formal de la firma. En este artículo se busca poner en consideración algunas propuestas teóricas a partir de evidencias empiricas, sobre las relaciones que surgen entre tales prácticas y los procesos gerenciales en las pequeñas y medianas empresas -Pymes-y sus resultados en términos de rentabilidad.

Para esto se hizo uso de metodologías de análisis estadístico de datos (metodologías multifactoriales) y econométricas (modelos de regresión) que permitieron, por un lado, resumir el número y complejidad de las variables consideradas a aquellas más representativas según su influencia en los resultados, principalmente en el ROA (return on assets) y en segundo lugar, indagar por las variables que mejor explican las diferencias en resultados financieros entre las empresas consideradas. Se pretende enriquecer el debate actual sobre la importancia de una gerencia articulada a las necesidades de las Pymes latinoamericanas, y la exploración de metodologías de intervención para la modernización de las actuales prácticas gerenciales en este tipo de organizaciones.

\section{ABSTRACT}

\section{$A$}

ny organizational result comes from a former managerial process, which needs an organizational structure that provides shape and action to communication and decisionmaking processes. In this paper, some theoretical proposals are shown based on empirical evidences about the relationship established on managerial pratices and processes in small and medium size companies, Pymes, and the results in terms of profitability.

For this reason, statistic data and econometric analysis methodologies were used which allowed, on one hand, to sum up the amount and complexity of considerable variables to those most representative according to their influence on given results, mainly on ROA (return on assets); and secondly, to llok for the best variables that explain the differences got in financial results among the selected companies. This study definetely enriches the current debate on the importanmce of having a management model according with the needs of Latinamerican Pymes, and the exploration of intervention methodologies to modernize current managerial practices in this type of organization.

\section{RESUMÉÉ}

$T$

out résultat entrepreneurial découle d'un processus administratif préalable ayant nécessité une structure organisationnelle fournissant la forme et l'action aux processus de communication et de décision, intervenant à leur tour à l'intérieur de la figure formelle de la signature. Dans cet article il s'agit de prendre en considération quelques propositions théoriques à partir de preuves empiriques sur les relations qui apparaissent entre certaines pratiques et processus administratifs petites et moyennes entreprises - PME et leurs résultats en termes de rentabilité. Pour cela il a été fait usage de méthodologies d'analyses statistiques de données (méthodologies plurifactorielles) et économétriques (modèles de régression) qui ont permis, d'une part, de réduire le nombre et la complexité des variables considérées les plus représentatives selon leur influence dans les résultats, principalement dans le ROA (return on assets) et, deuxièmement, d'étudier les variables qui expliquent mieux les différences des résultats financiers entre les entreprises considérées. On prétend enrichir le débat actuel sur l'importance d'une gestion articulée aux besoins des PME latino-americaines, et l'exploration de méthodologies d'intervention pour la modernisation des pratiques administratives actuelles dans ce type d'organisations. $\rightarrow$ Palabras claves

Modernización

Pymes

Rentabilidad

Componentes organizacionales

Sistémico

Gestión

Regresión

Multifactorial

\section{$\Rightarrow \quad$ Key words}

Modernization Pymes

Profitability

Organizational

components

Systemic

Management

Regression

Multifactorial

\section{$\Rightarrow \quad$ Mots clefs}

Modernisation

PME

Rentabilité

Composants

organisationnels

Systémique

Gestion

Régression

Plurifactorielle 


\section{INTRODUCCIÓN}

"Cuanto sabemos del mundo es sobre
la naturaleza de las teorías, y cuanto
la experiencia puede hacer es cambiar
estas teorías" (Hayek, 1952; pp. 143)
$\mathbf{E}$ ste estudio busca aportar un punto de vista teórico, a partir de evidencias empíricas, sobre las relaciones que surgen entre las prácticas gerenciales en las pequeñas y medianas empresas -PyMes- y sus resultados financieros.

Específicamente se explota el hecho de poder entender la gestión organizacional moderna como un conjunto de factores especializados en diferentes aspectos del ámbito empresarial, como por ejemplo: estrategia, mercados, cultura, finanzas, recursos humanos, etc., para establecer singularidades y rastrear efectos en la rentabilidad de las organizaciones.

La estructura conceptual, así como la metodología inductiva seguida por esta propuesta, tiene como leitmotiv la esencia del pensamiento de Hayek (1952), que se expone en la cita introductoria. Concretamente, se inicia tomando como base teórica el extenso marco que sustenta el desarrollo del Modelo de Modernización de la Gestión Organizacional -MMGO- (Pérez R.,et al. 2009), desarrollado por el grupo de investigación G3PyMes de la Universidad EAN, que busca generar un impacto significativo en la modernización de la gestión de las Pymes colombianas; a partir de esto, se busca contrastar evidencias empíricas recabadas a través de una muestra de ochenta de las ciento treinta y dos empresas analizadas por el MMGO entre los años 2005 y 2009, aportando finalmente una prototeoría que intenta conciliar los hallazgos en la realidad de las empresas, frente a las inferencias teóricas.

Concretamente se espera contrastar la hipótesis según la cual la modernización en términos de trabajar con mejores prácticas en la gestión empresarial de las Pyme se corresponde con un resultado positivo en la rentabilidad de la organización y que dicha modernización cumple con una estructura que permite identificar y afectar positivamente las variables gerenciales más críticas.

Aún cuando los hallazgos del presente esfuerzo resulten sólo en un ejercicio de la necesaria lúdica intelectual, no se desestima que estos puedan ayudar a concretar conceptos más refinados y exhaustivos, que aumenten nuestra comprensión de la empresa latinoamericana; o incluso pretender ir más lejos, gracias a la eventual emergencia de conceptos y categorías que influyen en la manera como afectamos nuestras organizaciones y entorno, quizás en el sentido que Ferraro, et al., (2003) lo consideran, al argüir "que las teorías de las ciencias sociales pueden convertirse en realidad debido a que forma los diseños institucionales y prácticas de gestión, así como las normas sociales y las expectativas sobre el comportamiento, creando de esta manera el comportamiento que predicen". En todo caso, es posible que los mayores logros estén en evidenciar con sus desaciertos y omisiones, campos de acción para eventuales esfuerzos de investigación. 


\section{BREVE PRESENTACIÓN CONCEPTUAL DEL MODELO DE MODERNIZACIÓN PARA LA GESTIÓN DE}

El MMGO trabaja de manera sistémica facilitando el establecimiento de prioridades para los proyectos de reconversión gerencial y productiva, proponiendo componentes o factores organizacionales que están compuestos por variables y descriptores con los que debe contar una empresa y definiendo las rutas que permiten optimizar dichos componentes. De estos, los seleccionados como de gran importancia o prioridad en un proceso de transformación de Pyme de sobrevivencia a Pyme innovadora, son: análisis y vigilancia del entorno, gestión del conocimiento, comunicación e información, mercadeo, comercio exterior (importaciones y exportaciones), gestión financiera, gestión de operaciones (producción y logística), gestión humana, estructura y cultura organizacional, gestión ambiental y responsabilidad social, dirección estratégica y asociatividad.

"El modelo como proceso, involucra varios aspectos o elementos claves de aprendizaje organizacional, por eso es sistémico: estadios o niveles (cuatro), situación actual o diagnóstico, definición de ruta, situación deseable o visión de futuro, capítulos, variables, descriptores, y curva de gestión. El proceso involucra a, exige cambios en, afecta a y se alimenta de gente con conocimiento (proceso de creación de conocimiento), estructura para favorecer el conocimiento, tecnologías para procesar y difundir el conocimiento y formas de medir el desempeño del conocimiento y la acción" (Pérez U, 2009. p. 52).

Los estadios se califican en cuartiles desde el primero (1) (0 a 25), segundo (2) (25-50), tercero (3) (50-75) y cuarto (4) (75-100). En el modelo, a partir del estadio 1 (figura 1), se define la ruta que se debe seguir para alcanzar estadios superiores, haciendo un recorrido por los diferentes componentes y bajo un exhaustivo análisis de las diferentes variables y descriptores que se encuentran teorizadas ${ }^{1}$, lo que permitirá encontrar la curva de gestión. Las guías e instrumentos diseñados por el G3PYMES ${ }^{2}$ de la Universidad EAN para la modernización de la gestión, permiten (Pérez,et al.,2009. P. 53 y 54):

Figura 1. Definición de la ruta hacia la innovación de la gestión de PyMES

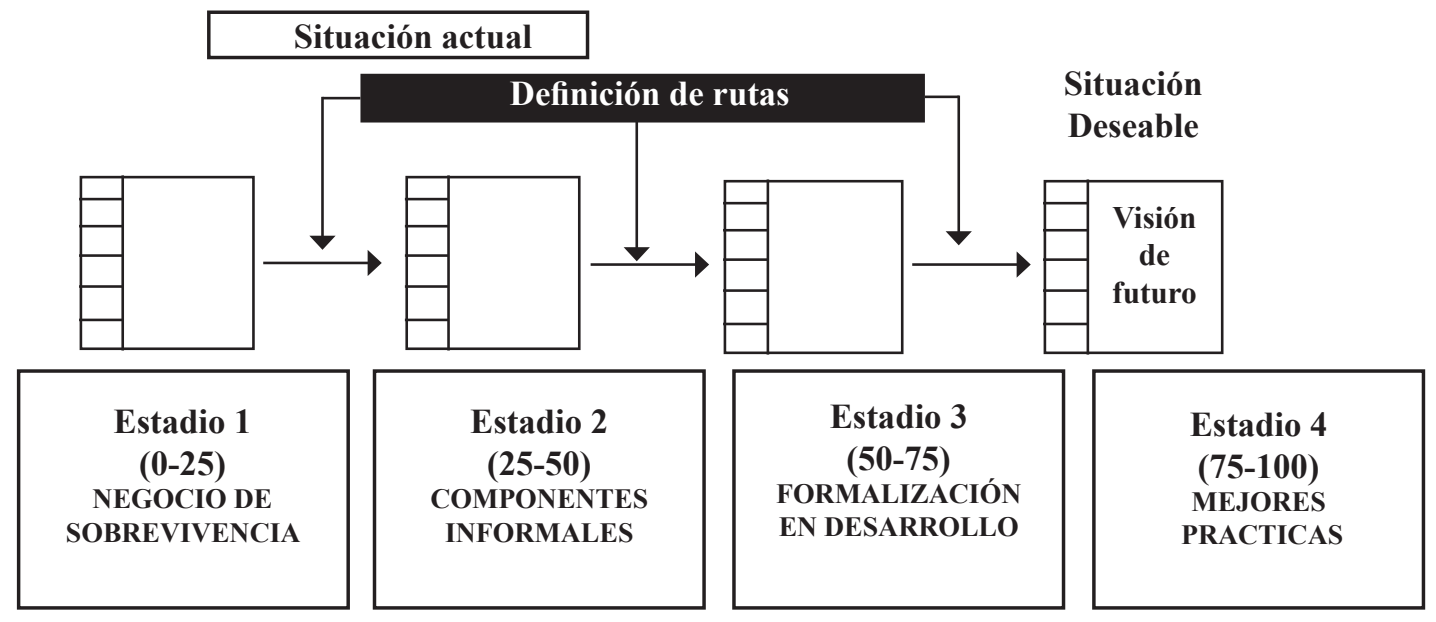

Fuente. Presentación MMGO. G3PyMES-2010

\footnotetext{
${ }^{1}$ Los documentos los puede adquirir en su versión 8 en la Universidad EAN - Vicerrectoría de investigación.

${ }^{2}$ Grupo de investigación en Gerencia de la gran, pequeña y mediana empresa (G3PYMES), Categoría A1 en Colciencias.
} 
$\Rightarrow$ Identificar la situación actual de la empresa con una visión integral, tomando en cuenta el nivel de desarrollo de cada uno de los elementos competitivos (estrategia, recursos humanos, finanzas, etc.).

$\Rightarrow$ Definir la visión de futuro a la cual se debe llegar.
$\Rightarrow$ Establecer estadios intermedios y las rutas con el fin de facilitar la mejora continua en la gestión de cada elemento y de la empresa como un todo.

A la fecha de este artículo se había aplicado el MMGO en cerca de 200 Pymes de diferentes ciudades en Colombia (Bogotá, Barranquilla, Bucaramanga, Villavicencio y Choco, entre otras).

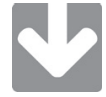

\section{APROXIMACIÓN AL PROBLEMA}

La aplicación del MMGO como herramienta de gestión estratégica para las Pymes se justifica en el comprobado efecto que sobre los resultados generales de las organizaciones tienen la aplicación de prácticas modernas de gestión. En este artículo no se discute la validez y el alcance de tal aserto $^{3}$, se toma como dado y constituye el primer supuesto de la presente propuesta teórica.

Si bien, suponer que las buenas prácticas en la gestión (moderna) de las organizaciones, resulte en beneficios más amplios para estas, parece algo trivial, el hecho es que en la práctica, no resulta tan evidente; en primer lugar, existe la necesidad de definir el concepto de gestión moderna y verificar si es posible contar con una especie de set de conceptos y prácticas, igualmente validos para las empresas de diferentes tamaños y actividades productivas y con culturas organizacionales heterogéneas, entre otras dificultades.

El hecho de que una determinada actividad empresarial resulte rentable, puede ser consecuencia de unas cuidadosas y sistemáticas prácticas de gestión, o por que se alcanzaron niveles de inversión y ventajas en el mercado que aseguran cierto control sobre los precios y la competencia, o más probable aún, que estos sistemas se autorefuercen en ambas direcciones. Estas y otras implicaciones de causalidad son relevantes y pueden conducir a diferentes y contrarias conclusiones, dependiendo de los aspectos que estemos tratando. No obstante, para los siguientes efectos siguientes, se supondrá que la causa yace inicialmente en el proceso gerencial (formal o implícito) del empresario, quien establece y sigue un plan de acción con la esperanza de obtener cierta rentabilidad.

Supondremos además que no hay problemas de coordinación ni límites a la información entre el principal y el agente, esto es, asumimos que los gerentes se comportan tal como esperan los propietarios que lo hagan, o en forma más sencilla, se asume simplemente que las empresas son dirigidas por sus propietarios quienes son personas racionales que privilegian la maximización del beneficio (en el largo plazo), que cualquier otro objetivo.

Considerando los anteriores supuestos generales, se pretende establecer una relación teórica entre los resultados de las organizaciones, medidos a partir del indicador financiero ROA ${ }^{4}$ (Return on Assets) y la gestión de la empresa en sus diferentes componentes o factores de gestión. Se seleccionó el ROA como la variable proxy a los resultados más generales de la empresa, dado que éste señala que tanto renta cada peso invertido en los activos totales de la empresa. En concreto tenemos que:

$$
R O A=F\left(x_{1}, \ldots, x_{n}\right) \quad[1]
$$

Es decir, los diferentes factores de la gestión de las organizaciones se combinan para dar como resultado un cierto nivel de rentabilidad, más específicamente, esta combinación supone una relación funcional de la siguiente forma:

\footnotetext{
$\overline{3}$ Para el lector interesado en las discusiones acerca de la relevancia de la gestión moderna en el resultado de las empresas se invita a consultar las referencias bibliográficas al final, así como la producción del grupo de investigación G3PyMes de la Vicerrectoría de Investigación de la Universidad EAN.

4 El indicador ROA es una variable financiera externa al MMGO, y es considerada como la variable de control fundamental del estudio, dado que esta variable resume suficientemente el comportamiento financiero y operacional de la organización.
} 


$$
\mathrm{F}\left(\mathrm{x}_{1}, \ldots, \mathrm{x}_{\mathrm{n}}\right)=\alpha+\sum_{\mathrm{i}}^{\mathrm{n}}=1 \beta_{\mathrm{i}} \mathrm{x}_{\mathrm{i}}, \quad \text { s.a. } \mathrm{x}_{\mathrm{i}} \geq 0, \mathrm{i}=1, \ldots, \mathrm{n}
$$

Donde la rentabilidad alcanzada por la organización es función de la agregación de los " $n$ " factores de gestión " $x$ ", que tienen lugar al interior de la empresa; que para el caso del MMGO cuenta con los 15 factores de evaluación (Pérez, et al., 2009), que lo compone. Los que además deben presentar valores positivos o nulos, es decir, deben tener sentido en la realidad.

Al igual que en la teoría económica de la producción, donde el nivel de producción depende de una cierta tecnología que combina los factores productivos de la manera más eficiente posible, la función [2, ] estaría implicando que la rentabilidad de la empresa, es función de cierta tecnología que combina eficientemente diferentes factores gerenciales.

Así como los factores de producción están sujetos a ciertas restricciones y supuestos dentro de la teoría del productor, donde estos factores son sujeto de rendimientos positivos pero decrecientes, cuando son considerados por separado. Los aquí denominados factores gerenciales, responden consecuentemente a ciertos criterios necesarios para la coherencia económica, como por ejemplo, la restricción observada en la ecuación [2] que implica que el dominio de la función se encuentrae entre los números positivos.

Otro supuestoes asumirque el aporte explicativo de un factor gerencial es variable, lo que indica un comportamiento no-lineal. Para ver esto, imaginemos que al interior de una organización el gerente decide incrementar rápidamente la inversión en el factor gerencial de planeación estratégica, dejando estables los demás. En este caso es fácil suponer que, una vez superada la capacidad instalada para la producción, así como la capacidad de respuesta de las demás unidades administrativas, cada peso adicional invertido en planeación estratégica, generará un impacto cada vez menor en la rentabilidad de la organización, inclusive llevando a disminuirla, lo que no esperaríamos en el caso de un gerente racional. Esto es, los factores gerenciales, tienen, al menos en ciertos niveles y de manera individual, un aporte positivo pero decreciente frente a la rentabilidad de la organización.
No obstante, la tecnología de gestión $\mathrm{f}(\mathrm{x})$ puede combinar los factores gerenciales de cierta forma para contrarrestar el efecto de saturación particular de cada factor, alcanzando niveles crecientes en la rentabilidad. O siguiendo con la analogía de la teoría de la producción, ciertas tecnologías de gestión podrían alcanzar niveles crecientes o constantes de escala en cuanto a la rentabilidad.

Una rápida revisión de los datos podría arrojar luces sobre este tipo de comportamientos, tanto en los resultados de rentabilidad de las empresas, como con respecto al comportamiento de los factores gerenciales. La figura 2 ordena una muestra de empresas diagnosticadas con el modelo MMGO para los cuales se obtuvieron rentabilidades superiores o iguales a cero.

Los datos se ajustan bastante bien $\left(\mathrm{R}^{2}>0,99\right)$ a una función cubica polinomial que comporta un incremento inicial rápido, seguido de un crecimiento lento y terminando de nuevo en forma acelerada. Esta distribución bien ajustada de las empresas según su rentabilidad tal vez no parezca muy reveladora, pero al observar la distribución de las empresas según sus niveles de gestión en cada componente evaluado por el MMGO, encontramos el mismo tipo de relación.

El hecho de que las empresas sigan una trayectoria respecto de los factores gerenciales similar a la de la rentabilidad ROA, podría estar indicando ciertos procesos de agregación de los esfuerzos en gestión, que se traducen en similares comportamientos en los niveles de rentabilidad de la empresa. No obstante, este comportamiento de los datos puede resultar de una causa externa no vinculante, por lo que será necesario verificar estadísticamente dicha hipótesis. Esta tarea se deja para más adelante, una vez se hayan tratado los asuntos de procedimiento metodológico, necesarios para hacer compatibles los datos aportados por el MMGO y el ROA. 
Figura 2. Distribución de las empresas según ROA

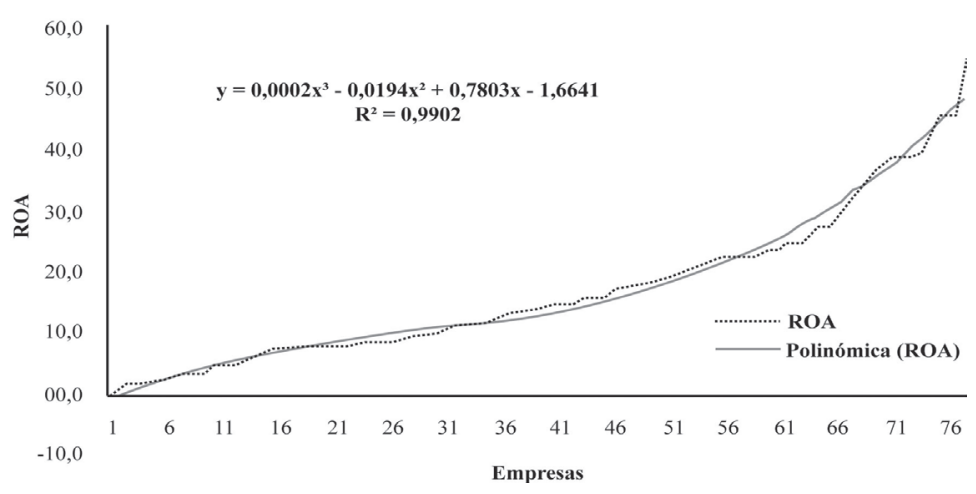

Fuente. Cálculos propios con base en datos MMGO, Vicerrectoría de Investigación, Universidad EAN, 2009.

Figura 3. Distribución de las empresas según nivel alcanzado en algunos factores gerenciales evaluados por el MMGO

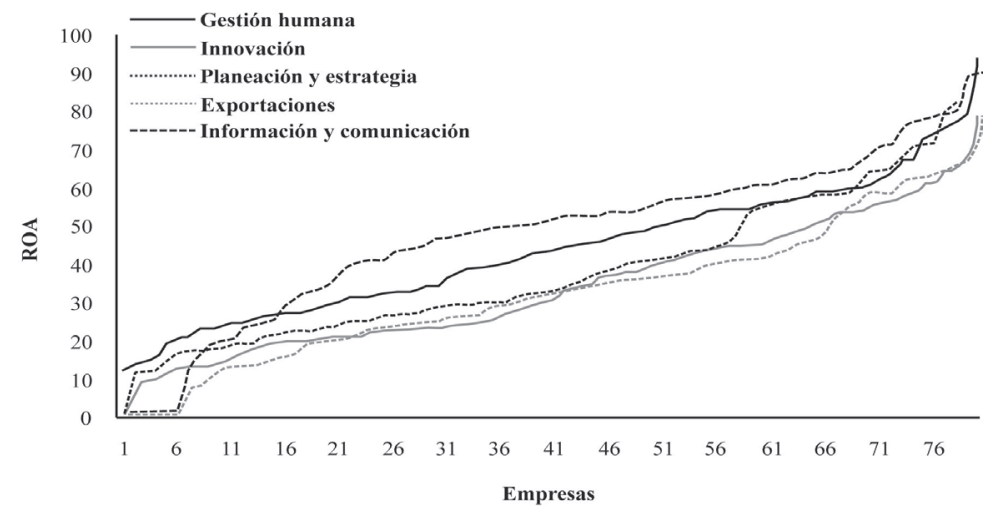

Fuente. Cálculos propios con base en datos MMGO, Vicerrectoría de Investigación, Universidad EAN, 2009.

De momento, supongamos sin mayores demostraciones, que la ecuación [2] representa aceptablemente la relación entre los factores gerenciales y el ROA. Si quisiéramos analizar el efecto que cada factor gerencial, digamos, la gestión humana, tiene unilateralmente sobre la rentabilidad de la organización, sería necesario evaluar su aporte marginal, en cuyo caso estaría definido como sigue:

$$
\frac{\partial F\left(x_{1}, \ldots, x_{n}\right)}{\partial \dot{x}}=\sum_{i=1}^{n} \frac{\partial B_{i} x_{i}}{\partial x_{i}} \quad i=1, \ldots, n
$$

La trayectoria espacial que siguen las " $n$ " ecuaciones marginales derivadas de la función 2 , describirían una trayectoria convexa, donde los efectos de una mayor inversión en, digamos, gestión humana, tiene un efecto en la rentabilidad cada vez menor, alcanzando un mínimo a partir del cual se incrementa su aporte nuevamente. Este comportamiento de los factores gerenciales frente a la escala o intensidad de su utilización, y su efecto en la rentabilidad organizacional implica cierto punto crítico de mínimo efecto, donde los niveles de inversión a la izquierda de este son decrecientes.

Suponiendo esto, podríamos esperar que un gerente racional que se enfrente a este modelo, estuviera dispuesto a soportar que sus esfuerzos en gestión sean cada vez menos efectivos frente a los resultados en la rentabilidad, con la certeza que dentro de cierto margen, la inversión adicional en cualquier factor gerencial, será cada vez más rentable. Por desgracia, en la práctica, las rentabilidades de una actividad productiva no crecen at infinitum; existen límites de mercado, tecnológicos o de otra índole, diferentes a la gestión de la organización, que imponen cotas a los resultados financieros de las empresas. 
Antes de seguir con los constructos de una propuesta teórica, y de intentar probar la coherencia y validez de nuestras hipótesis frente a los datos, es conveniente observar la metodología de adecuación y depuración de la información-insumo, obtenida de la muestra de trabajo.

\section{El DESARROLLO de ESTA INUESTIGACIÓN}

Los datos necesarios para el presente ejercicio provienen de una muestra de 80 pequeñas y medianas empresas de las cerca de doscientos a las cuales se les ha aplicado el MMGO, ubicadas en la ciudad de Bogotá y su zona de influencia económica, diagnosticadas mediante el modelo y sus correspondientes estados financieros con información necesaria para el cálculo del ROA.

Como se mencionó anteriormente el MMGO examina 15 factores gerenciales con el objeto de ubicar a la empresa en uno de cuatro estadios de modernización posibles; donde el Estadio 4 corresponde a la empresa tipo de clase mundial que lleva a cabo las mejores prácticas en gestión

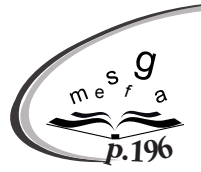

y esta conectadas a los circuitos internacionales de comercio, en tanto que el Estadio 1 corresponde a la empresa con sistemas de gestión informales poco eficientes y de adopción empírica, que operan en mercados limitados y con una estructura administrativa y jerárquica disfuncional. Los Estadios 2 y 3 representan el camino intermedio entre estos dos estadios extremos.

La ubicación de una empresa en uno u otro estadio, depende del promedio alcanzado en cada uno de los 15 factores gerenciales, los que a su vez, se componen de varios descriptores que, basados en la más reciente teoría gerencial, disponen las características de cada uno de los cuatro estadios, siendo estos últimos los que objetivamente evalúa el gerente de la empresa y/o el responsable del proceso, junto a un consultor experto en la metodología MMGO. Esta disposición de evaluación resulta en una serie de variables de carácter ordinal por descriptivo, que se agregan para dar un promedio por factor gerencial, que dependiendo del cuartíl alcanzado, asigna de nuevo uno de cuatro estadios al factor. El proceso se repite para dar por último un estadio general a la empresa.
En contraste, la información de rentabilidad (ROA) es una variable métrica, que puede tomar valores tanto negativos como positivos, sin restricción y plantea un primer inconveniente a las pretensiones del presente ejercicio, pues en principio supone explicar un comportamiento escalar (ROA), mediante una serie de variables cualitativas ordinales (15 factores gerenciales).

En segundo lugar, las pretensiones principalmente teóricas, pero también de aplicación empírica, obligan a considerar el principio de parsimonia ${ }^{5}$, el cual reconoce la imposibilidad de cualquier modelo para representar completamente la realidad, además de que este esfuerzo carecería de utilidad práctica. "En cualquier construcción de modelos es inevitable hacer abstracciones o simplificación en alguna medida" (Gujarati, 1997; pp.444). Es decir, esperamos que nuestra propuesta teórica sea lo más efectiva posible, al explicar la mayor cantidad de fenómenos con la menor cantidad de variables.

Estudios precedentes (Ortiz,2008), que intentan identificar estructuras y relaciones en las empresas evaluadas por el MMGO, dentro de un enfoque de la interdependencia, esto es, donde ninguna variable destaca como dependiente o explicativa principal, teniendo en principio la misma importancia y tratamiento relativo y por lo tanto, independiente de teorías subyacentes. Permitió, entre otros, generar insumos necesarios para superar parte de los inconvenientes mencionados: la combinación de variables métricas y cualitativas, y establecer el principio de parsimonia; que ahora es necesario representar en un enfoque metodológico de la dependencia capaz de generar pronósticos e inferencias.

Se aborda primero el asunto de la simplificación del problema. En Ortiz(2008) se emplearon metodologías estadísticas conocidas como de reducción de las dimensiones

\footnotetext{
$\overline{5}$ Este principio también se conoce como el de la hoja de afeitar de Occam, el cual establece que un modelo se debe mantener tan simple como sea posible.
} 
con variables cualitativas, o modelos de correspondencias múltiples, que tienen como objetivo establecer relaciones entre variables no métricas (cualitativas) para obtener un mapa perceptual que ponga de manifiesto esta asociación en modo gráfico, y obtener así un número menor de nuevas variables ficticias (dimensiones) que representan las originales con la mínima pérdida de información. (Pérez C., 2005; pp. 533).

En concreto se obtuvieron dos nuevas variables o dimensiones que resumen en forma eficiente lo ocurrido al interior del grupo de empresas en términos de variabilidad y según los factores gerenciales que las describen. Las tablas siguientes resumen estos resultados.

Tabla 1. Ranking de las medidas de discriminación. Dimensiones 1 y 2

\begin{tabular}{|c|c|c|c|c|c|}
\hline \multicolumn{2}{|c|}{ Dimensión 1} & \multirow[b]{2}{*}{0,640} & \multicolumn{2}{|r|}{ Dimensión 2} & \multirow[b]{2}{*}{0,463} \\
\hline 1. & $\begin{array}{l}\text { Planeación y } \\
\text { Estrategia }\end{array}$ & & 1. & Asociatividad & \\
\hline 2. & $\begin{array}{l}\text { Gestión } \\
\text { Humana }\end{array}$ & 0,578 & 2. & $\begin{array}{l}\text { Comunicación e } \\
\text { Información }\end{array}$ & 0,435 \\
\hline 3. & $\begin{array}{l}\text { Comunicación e } \\
\text { Información }\end{array}$ & 0,561 & 3. & $\begin{array}{l}\text { Cultura } \\
\text { Organizacional }\end{array}$ & 0,38 \\
\hline 4. & $\begin{array}{l}\text { Innovación y } \\
\text { Conocimiento }\end{array}$ & 0,537 & 4. & $\begin{array}{l}\text { Estructura } \\
\text { Organizacional }\end{array}$ & 0,363 \\
\hline 5. & $\begin{array}{l}\text { Estructura } \\
\text { Organizacional }\end{array}$ & 0,527 & 5. & Gestión Humana & 0,341 \\
\hline 6. & $\begin{array}{l}\text { Cultura } \\
\text { Organizacional }\end{array}$ & 0,493 & 6. & $\begin{array}{l}\text { Innovación y } \\
\text { Conocimiento }\end{array}$ & 0,317 \\
\hline 7. & Finanzas & 0,474 & 7. & $\begin{array}{l}\text { Planeación y } \\
\text { Estrategia }\end{array}$ & 0,278 \\
\hline 8. & $\begin{array}{l}\text { Gestión de } \\
\text { Mercados }\end{array}$ & 0,464 & 8. & Finanzas & 0,259 \\
\hline 9. & Asociatividad & 0,394 & 9. & Logística & 0,248 \\
\hline 10. & $\begin{array}{l}\text { Gestión de } \\
\text { Producción }\end{array}$ & 0,371 & 10. & $\begin{array}{l}\text { Gestión de } \\
\text { Producción }\end{array}$ & 0,108 \\
\hline 11. & Logística & 0,367 & 11. & Importaciones & 0,096 \\
\hline 12. & Exportaciones & 0,293 & 12. & $\begin{array}{l}\text { Gestión de } \\
\text { Mercados }\end{array}$ & 0,035 \\
\hline 13. & Importaciones & 0,243 & 13. & Exportaciones & 0,029 \\
\hline 14. & Medio ambiente & 0,220 & 14. & Medio ambiente & 0,086 \\
\hline \multicolumn{2}{|c|}{$\%$ de la varianza } & 44,021 & \multicolumn{2}{|c|}{$\%$ de la varianza } & 24,556 \\
\hline
\end{tabular}

Fuente. Ortíz, 2008: Metodología para evaluación del impacto de la gestión en los resultados financieros de las organizaciones.

La importancia que cada variable comporta está dada por el porcentaje de varianza que explica; en el caso de la dimensión 1 se observa (última fila) un porcentaje de $44 \%$ en la varianza explicada, y en la dimensión 2, casi una cuarta parte del total.

Los valores de las medidas de discriminación de cada factor gerencial, indican la importancia que estos tienen de la dimensión correspondiente, así, planeación y estrategia, gestión humana y comunicación e información son, en su orden, los tres factores más importantes en la dimensión 1, en tanto que en la 2 son: asociatividad, comunicación e información y cultura organizacional.

Las medidas de discriminación de los factores, además de indicar la importancia de estos en las nuevas variables o dimensiones, permite identificar cuales discriminan mejor las empresas según su perfil dentro de cada dimensión, esto es, aquellos que caracterizan con mayor fuerza las empresas asociadas a los factores principales de la 
dimensión respectiva. La tabla 2 enlista las diferencias en estas medidas por factor, evidenciando que el factor gestión de mercados es la variable que más discrimina las empresas caracterizadas por altos valores en los factores más importantes de la dimensión 1, en tanto que asociatividad es el factor que mejor discrimina las empresas identificadas con los factores más importantes de la dimensión 2.

Tabla 2. Distancia entrre las medidas de discriminación de los factores. (Dimensión 1-2)

\begin{tabular}{|c|c|c|}
\hline Factor & & $\begin{array}{c}\text { Distancia Dimensión } \\
1 \text { a } 2 \\
\end{array}$ \\
\hline 1. & Gestión de Mercados & 0,429 \\
\hline 2. & Planeación y Estrategia & 0,362 \\
\hline 3. & Exportaciones & 0,264 \\
\hline 4. & Gestión de Producción & 0,263 \\
\hline 5. & Gestión Humana & 0,237 \\
\hline 6. & Innovación y Conocimiento & 0,220 \\
\hline 7. & Finanzas & 0,215 \\
\hline 8. & Estructura Organizacional & 0,164 \\
\hline 9. & Exportaciones & 0,147 \\
\hline 10. & Medio Ambiente & 0,134 \\
\hline 11. & $\begin{array}{l}\text { Comunicación e } \\
\text { Información }\end{array}$ & 0,126 \\
\hline 12. & Logística & 0,119 \\
\hline 13. & Cultura Organizacional & 0,113 \\
\hline 14. & Asociatividad & $-0,069$ \\
\hline
\end{tabular}

Fuente. Ortíz, 2008: Metodología para evaluación del impacto de la gestión en los resultados financieros de las organizaciones.

De esta manera, la metodología de reducción de las dimensiones del modelo de correspondencias múltiples, permite establecer un ranking de los factores gerenciales, que resultan más relevantes a la hora de explicar las diferencias (varianza) que existen al interior de las empresas y entre estas, estableciendo variables candidatas, estadísticamente válidas, ha ser incorporadas en modelo teórico propuesto.

Pero las ventajas no sólo están en la identificación del núcleo de variables principales, sino que, como subproducto de la mencionada metodología, se obtienen puntuaciones para cada factor gerencial de cada empresa, generando así nuevas variables métricas, continuas, y que adicionalmente, superan cualquier problema de multicolinealidad que pudieran presentar las variables originales, lo que es probable dadas las restricciones en el acceso a la población de empresas objeto del muestreo.

Estas nuevas puntuaciones por factor, que constituyen variables proxy de las originales, permiten ahora plantear relaciones entre variables métricas continuas y ampliar las posibilidades de tratamiento empírico de la propuesta teórica más allá de los ajustes de elección discreta de los modelos lineales de probabilidad (Probit y Logit), incluyendo, la no linealidad, al menos en las variables, lo que nos resultará útil más adelante.

De esta manera contamos con una muestra de 80 empresas con valores válidos en las variables que se enlistan, como se observa en la tabla 3 . 
Tabla 3. Lista de variables incluidas

\begin{tabular}{|l|l|}
\hline \multicolumn{1}{|c|}{ Etiqueta } & \multicolumn{1}{c|}{ Nombre } \\
\hline ROA & ROA \\
\hline CIIU & Actividad Productiva \\
\hline Estrategia & Planeación y Estrategia \\
\hline Mercados & Gestión de Mercados \\
\hline Cultura & Cultura Organizacional \\
\hline Estructura & Estructura Organizacional \\
\hline Producción & Gestión de Producción \\
\hline Finanzas & Finanzas \\
\hline Humana & Gestión Humana \\
\hline Exportaciones & Exportaciones \\
\hline Importaciones & Importaciones \\
\hline Logística & Logística \\
\hline Asociatividad & Asociatividad \\
\hline Información & Comunicación e información \\
\hline Innovación & Innovación y conocimiento \\
\hline Ambiente & Medio ambiente \\
\hline
\end{tabular}

Fuente. Ortíz, 2008: Metodología para evaluación del impacto de la gestión en los resultados financieros de las organizaciones.

Como nota aclaratoria se indica que en este trabajo se emplea indiferentemente los términos de intensidad o nivel de inversión, de los factores gerenciales, para hacer referencia al grado de utilización de estos al interior de la organización. En concreto se supone que un estadio más avanzado o modernizado implica un esfuerzo mayor en inversión tanto de recursos físicos como financieros y humanos.

\section{Resultados}

Si bien los modelos estadísticos de la interdependencia, como los empleados en Ortiz (2008) y Pérez R. et al., (2009), permiten describir fenómenos dependiendo de los atributos que los componen en forma multidimensional, resultando muy adecuados para la comprensión estructural de un problema de estudio y correspondiente clasificación de individuos (empresas) de manera eficiente, su capacidad de inferencia y pronóstico resulta limitada o nula. Esto es así, porque, entre otras, la falta de verificación en la direccionalidad entre causas y efectos limita las posibilidades de establecer trayectorias analíticas.

No obstante las limitaciones de inferencia y pronóstico de los modelos de la interdependencia no deberían buscarse en sus características matemáticas o estadísticas, tanto como en la teoría que subyace en su metodología; en este tipo de modelos se analizan los datos per se (Pérez C., 2005) y son estos (los datos) el objeto de las indagaciones teóricas, por lo que no nos debería extrañar sus límites para avanzar hacia posiciones adelante o atrás, que intenten observar algún proceso de evolución en un sistema más complejo.

Con lo anterior se quiere justificar lo que desde la literatura de modelación econométrica, resulta elemental. Sí queremos contar con un modelo que nos permita inferir y pronosticar sobre una determinada población, es necesario establecer en primer lugar, ciertos niveles de dependencia, definiendo al menos variables de control y de estado. Que una variable se considere de control, explicada, regresada o endógena y otras de estado, explicadores, regresores o exógenas, depende específicamente de la teoría seleccionada o desarrollada para explicar el fenómeno general, a partir de la interacción y condiciones de dependencia de las variables involucradas.

Es usual, que en estudios de carácter socioeconómico se establezcan modelos que se soportan en una teoría que busca explicar el fenómeno objeto de atención, definiendo así qué explica qué dentro de la modelación formal y empírica. Entre tanto, en estudios que tienen como objeto los fenómenos gerenciales de las organizaciones, se recurre con frecuencia a indagaciones derivadas de metodologías basadas en el análisis detallado de los datos, 
de las relaciones y de las estructuras que estos puedan mantener, para luego deducir conclusiones sobre el fenómeno de estudio; esto es lo que sucede por ejemplo en metodologías basadas en estudios de caso o en grandes encuestas. Tanto uno como otro enfoque son igualmente lícitos, y su utilidad dependerá del tipo de resultados a los que se quiera llegar, como de la inclinación metodológica del investigador.

En este estudio se intenta desarrollar una metodología que permita inferir y/o pronosticar sobre los resultados en

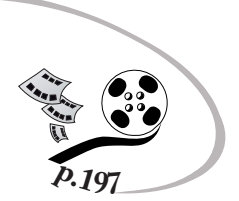
rentabilidad de las Pymes a partir de sus atributos en materia de gestión organizacional, por lo que es necesario establecer un enfoque de la dependencia y desde luego, contar con una teoría que oriente la consecuente modelación econométrica, necesaria para dar alcance a los objetivos establecidos.

Entonces, ¿qué teoría debe soportar las indagaciones que se pretenden en este estudio? Las opciones son prolíficas, por ejemplo, el diseño del MMGO (Pérez, R. et. al, 2009), cuenta con una serie de teorías de reciente generación que sustentan cada uno de los descriptores que componen cada factor gerencial (Pérez, et al., 2009). No obstante, aquí no se recurrirá a marcos teóricos exhaustivos y como premisa se atenderá al principio de parsimonia arriba mencionado, y se establecerá una prototeoría basada en la concepción de la empresa como unidad transformadora y generadora de valor.

La empresa, como función, establece una tecnología para combinar ciertos factores productivos y transformarlos en bienes o servicios valiosos para la sociedad. En la literatura económica y de gerencia se suele considerar como factores principales el capital (k), trabajo (l) y el conocimiento $(\mathrm{w})$, entre otros, donde por ejemplo, el factor capital incluye tanto los insumos necesarios en la fabricación de un producto, como las máquinas y la planta $\mathrm{y}$ otros recursos. Sabemos que la inclusión de un insumo en el proceso productivo, digamos la tinta en la elaboración de libros, es el resultado de un proceso de compra realizado por la empresa (quizás por un departamento de compras) en un mercado de materias primas, que involucra la consideración de una enorme cantidad de variables como precios, stock, pedidos realizados, tasas de cambio, acuerdos comerciales, competencia, calidad, etc., que precisan ser gerenciadas y gestionadas. Similar complejidad señala el factor trabajo: un departamento de personal debe contratar el personal necesario para el proceso productivo, negociar una remuneración y establecer incentivos para que sus empleados más valiosos y productivos no marchen a la competencia, que cuenten con las cualificaciones necesarias para su adecuado desempeño, y todo esto implica de nuevo un proceso de gestión sobre el factor humano.

Podríamos establecer para cada proceso importante de la empresa, una actividad gerencial que implica la capacidad de personas reales de comunicarse e interactuar con otras personas reales. En esencia, el asunto de la empresa, al igual que cualquier otro de la sociedad, es un asunto de redes y nodos de comunicación donde tienen lugar las decisiones.

Así que los núcleos de gestión como los definidos por el MMGO, resultan ser muy potentes para sintetizar complejos procesos humanos enmarcados dentro de lógicas productivas y comerciales de las empresas. Este enfoque, adicionalmente, permite colocar el conocimiento (tácito o explicito) como elemento primigenio en cada factor gerencial; es el conocimiento lo que valida al individuogerente a conectarse efectivamente en un proceso de comunicación y negociación.

Considerando lo anterior, se debería justificar definir la empresa como una función $\mathrm{F}\left(\mathrm{x}_{1}, \ldots, \mathrm{x}_{\mathrm{n}}\right)$ que integra "n" factores gerenciales para alcanzar de la manera más eficiente posible los objetivos productivos, comerciales y de beneficio que se haya propuesto. Para ser más específicos, se considera que la tecnología empleada adiciona los factores gerenciales en la cantidad (n) y en la intensidad $(\beta)$ que requiera el producto o servicio que se esté desarrollando. Lo que se expresa en la ecuación [2] que se trascribe a continuación.

$$
F\left(x_{1}, \ldots, x_{n}\right)=\alpha+\sum_{i=1}^{n} \beta_{i} x_{i}, \text { s.a. } x_{i} \geq 0, \quad i=1, \ldots, n .
$$

Establecido el modelo teórico, podemos plantear entonces el siguiente modelo econométrico estocástico: 


$$
\mathrm{ROA}_{\mathrm{t}}=\beta_{\mathrm{o}}+\sum_{\mathrm{i}=1}^{\mathrm{n}} \beta_{\mathrm{i}} \mathrm{x}_{\mathrm{i}}+\mu \quad \mathrm{t}=1,2,3 \ldots \mathrm{T}
$$

En el planteamiento de la ecuación [3] se asume implícitamente que el grado de éxito de una empresa, o efectividad de su tecnología de gestión organizacional, se refleja en su rentabilidad. El subíndice $t$ indica las observaciones realizadas o el tamaño de la muestra.

Dado que las variables $\mathrm{x}$ correspondientes a los factores gerenciales son tomadas de las puntuaciones arrojadas por la aplicación de metodologías multifactoriales a la muestra categórica original (Ortiz, 2008), se sabe que no existen problemas de multicolinalidad y procedemos a desarrollar una metodología mínimo-cuadrática para la estimación del modelo [4].

Antes de introducir los datos al modelo, se suavizaron las series de datos, para lo cual se calcularon medias móviles, con rango de ocho observaciones.

La especificación inicial del modelo se estableció con los primeros 5 factores gerenciales más relevantes en términos de su capacidad de discriminación en la dimensión 1 (ver tabla 1): planeación y estrategia (estrategia), gestión humana (humana), comunicación e información (información), innovación y conocimiento (innovación), Estructura organizacional (estructura) y una variable adicional, Exportaciones, que se caracteriza por tener una mínima influencia en las dos dimensiones establecidas, pero que tiene una importante capacidad de discriminar las empresas que mejor se asocian a una u otra dimensión (ver Tabla 2).
Esto es:

$$
\begin{gathered}
\left.\mathrm{ROA}=\beta_{0}+\beta_{1} \text { (Estrategia) }+\beta_{2} \text { (Humana }\right)+\beta_{3} \\
\left.(\text { Información })+\beta_{4} \text { (Innovación }\right)+\beta_{5} \\
\text { (Estructura })+\beta_{6}(\text { Exportaciones })+\mu
\end{gathered}
$$

Este modelo demostró tener un buen ajuste a los $₫ 4$ At $\$ \mathrm{~s}$ $\left(\mathrm{R}^{2}=0,952\right)$, y sin embargo, los coeficientes $\beta$ resultaron ser poco significativos, presentando alta probabilidad de llegar a ser cero $(>0,50)$.

En virtud de este resultado, es factible deducir que la especificación del modelo puede estar incorporando demasiadas variables. Para probar esto, se corrió el mismo modelo empleando un método de eliminación hacia atrás, que implica excluir la variable con menor correlación parcial con la variable dependiente, hasta que queden únicamente aquellas cuyos coeficientes tengan una probabilidad de ser cero menor al $10 \%$. Las salidas de este procedimiento se presentan a continuación; en la primera columna se enlistan las variables incluidas en cada corrida del modelo, en la segunda y tercera columna los coeficientes y sus estadísticos de error típico, y en las siguientes columnas los coeficientes estandarizados, las probabilidades " $t$ " y el grado de significancia de cada coeficiente.

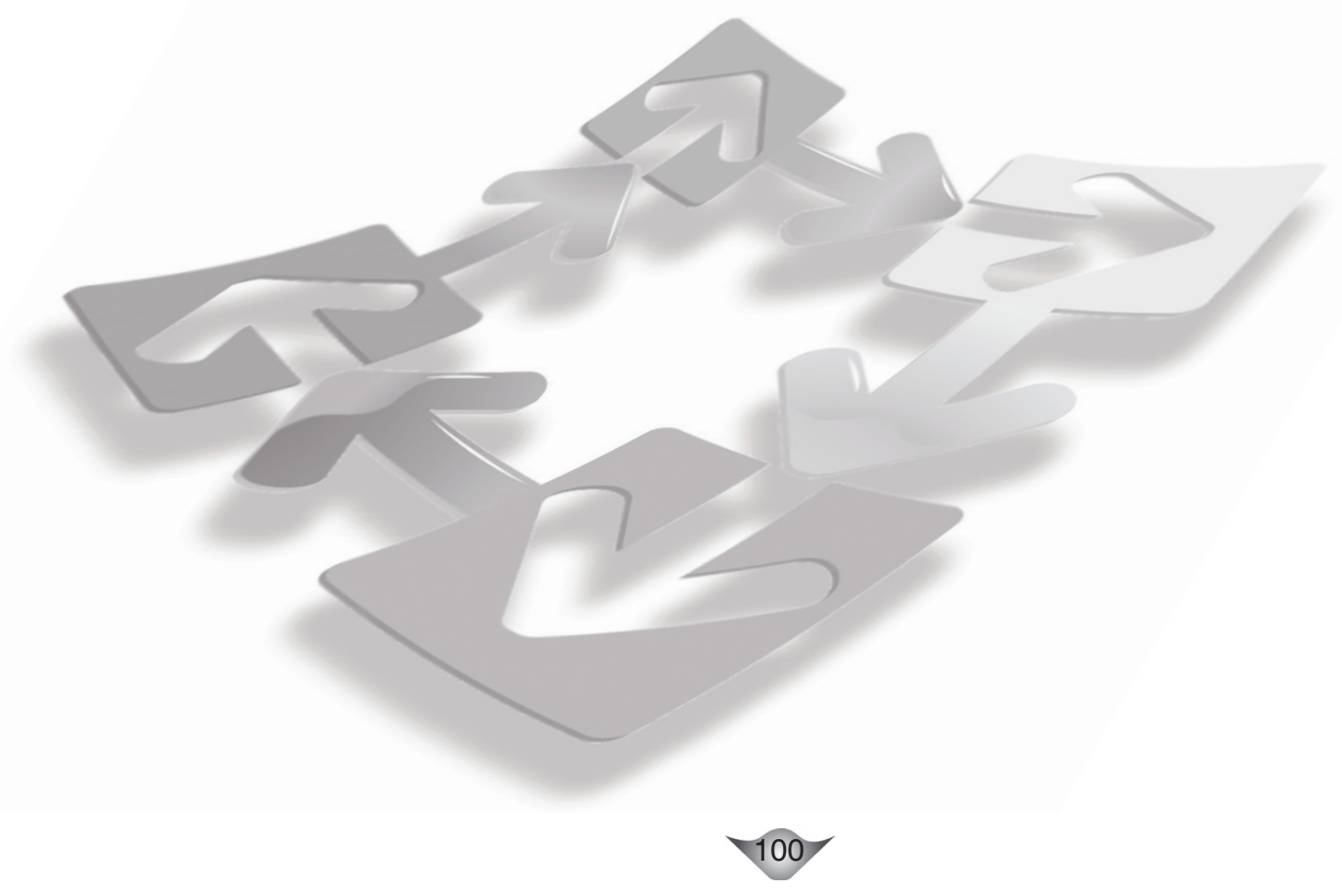


Tabla 4. Coeficientes modelos de regresión según variables eliminadas

\begin{tabular}{|c|c|c|c|c|c|c|}
\hline \multicolumn{2}{|r|}{ Modelo } & \multicolumn{5}{|c|}{ Coeficientes estandarizados } \\
\hline & & $\mathrm{B}$ & Error típ. & Beta & $\mathbf{T}$ & Sig. \\
\hline \multirow[t]{7}{*}{1} & (Constante) & $-104,278$ & 40,961 & & $-2,546$ & 084 \\
\hline & Estrategia &, 187 & 1,357 & 076 &, 138 & 899 \\
\hline & Estructura & ,957 & ,940 & ,392 & 1,017 & ,384 \\
\hline & Humana &, 124 & 647 & 061 & ,192 & ,860 \\
\hline & Información & 1,025 & 1,003 & ,359 & 1,022 & ,382 \\
\hline & Innovación &, 524 & ,980 &, 187 &, 535 & 630 \\
\hline & Exportaciones & ,090 & 601 & 033 &, 150 &, 890 \\
\hline \multirow[t]{6}{*}{2} & (Constante) & $-106,847$ & 31,682 & & $-3,373$ & ,028 \\
\hline & Estructura & ,982 & ,802 & ,402 & 1,225 & ,288 \\
\hline & Humana &, 170 & ,482 & ,084 &, 352 & ,742 \\
\hline & Información & 1,113 & 674 & 390 & 1,652 &, 174 \\
\hline & Innovación & ,585 & ,761 & ,208 & ,768 &, 485 \\
\hline & Exportaciones & ,097 &, 520 & 036 & , 186 &, 861 \\
\hline \multirow[t]{5}{*}{3} & (Constante) & $-102,574$ & 19,603 & & $-5,233$ & ,003 \\
\hline & Estructura & ,969 & ,717 & ,397 & 1,351 & ,235 \\
\hline & Humana &, 150 &, 423 &, 074 & ,356 &, 737 \\
\hline & Información & 1,141 &, 590 &, 400 & 1,934 &, 111 \\
\hline & Innovación &, 544 &, 655 & ,194 &, 831 & ,444 \\
\hline \multirow[t]{4}{*}{4} & (Constante) & $-101,134$ & 17,728 & & $-5,705$ & 001 \\
\hline & Estructura & 1,092 &, 581 & ,447 & 1,878 & , 109 \\
\hline & Información & 1,108 &, 538 & ,388 & 2,057 &, 085 \\
\hline & Innovación &, 589 &, 594 & ,210 & ,991 &, 360 \\
\hline \multirow[t]{3}{*}{5} & (Constante) & $-100,864$ & 17,704 & & $-5,697$ & ,001 \\
\hline & Estructura & 1,449 &, 455 & ,594 & 3,184 &, 015 \\
\hline & Información & 1,186 &, 532 & ,416 & 2,230 & ,061 \\
\hline
\end{tabular}

Fuente. Cálculos propios con base en datos Ortiz, 2008. Salidas SPSS.

El mejor ajuste, según grados de significancia menores al $10 \%$, se alcanza en el quinto modelo, teniendo como variables explicativas: estructura $(0,015)$ e información $(0,061)$, cuyos coeficientes señalan un buen comportamiento (menores al 7\%). No obstante, el modelo 4 indica una especificación razonable, si estuviéramos dispues-tos a enfrentar una probabilidad de equivocarnos en 36 de cada 100 veces que consideremos el factor gerencial de innovación y conocimiento, adicionalmente, la observación de significación conjunta de los tres parámetros ( $\mathrm{p}$-valor del contraste de la F) resulta altamente significativo a un $95 \%$ de confianza.

Asumir tal relajación de los estadísticos podría ser comprensible en virtud a la variedad de actividades productivas, servicios y comerciales, a las que se dedican las Pymes encuestadas. La rentabilidad no sólo depende de que también se gerencia una empresa, sino también en qué sector económico se encuentre (Hall, 1983. P. 231 a 233); la gerencia de una empresa productora de molinos de pulpa para papel pudiera ser excelente, y aún así, obtener una rentabilidad inferior a una empresa explotadora de mineral de carbón pesimamente administrada.

Como se señala más adelante, el realizar regresiones ponderadas según la clasificación industrial CIIU, demostró mejorar tanto el ajuste del modelo, como los estimadores, aún cuando desafortunadamente, la muestra de trabajo no contenía una frecuencia suficiente por actividad industrial, como para realizar estimaciones separadas por industrias, 
pero se deja como recomendación para posteriores estudios, una vez se cuente con una muestra representativa por sector.

Volviendo a la especificación del modelo, consideremos entonces la quinta opción, donde tenemos que:

ROA=-101,134+1,108(Información) $+1,092$

(Estructura) +0,589(Innovación)
Esta especificación del modelo indica un buen ajuste con un $\mathrm{R}$ cuadrado (corregido) elevado $(0,855)$. El valor del estadístico Durbin-Watson cercano a 2 certifica la ausencia de autocorrelación. Así mismo, la tabla ANOVA indica un p-valor del contraste de la $\mathrm{F}$ para la significación conjunta de los parámetros estimados, menor a 0,05 , indicando que hay alta significatividad conjunta de los coeficientes del modelo. En las dos tablas siguientes se resumen los principales estadísticos y resultados.

Tabla 5. Ajuste y bondad del modelo de regresión No.5.

\begin{tabular}{|c|c|c|c|c|c|c|c|c|c|}
\hline \multicolumn{9}{|c|}{ Estadísticos de cambio } & \multirow{2}{*}{$\begin{array}{l}\text { Durbin } \\
\text { Watson }\end{array}$} \\
\hline $\mathbf{R}$ & $\begin{array}{c}\mathbf{R} \\
\text { cuadrado }\end{array}$ & $\begin{array}{l}\mathbf{R} \text { cuadrado } \\
\text { corregida }\end{array}$ & $\begin{array}{c}\text { Error típ. } \\
\text { de la } \\
\text { estimación }\end{array}$ & $\begin{array}{c}\text { Sig. del } \\
\text { cambio en } F\end{array}$ & $\begin{array}{l}\text { Cambio en } \\
R \text { cuadrado }\end{array}$ & $\begin{array}{c}\text { Cambio } \\
\text { en } \mathbf{F}\end{array}$ & gl1 & gl2 & \\
\hline ,950(a) & ,903 & 855 & 5,77071 & ,903 & 18,630 & 3 & 6 &, 002 & 2,083 \\
\hline
\end{tabular}

a. Variables predictoras: (constante), innovación, información, estructura.

b. Variable dependiente: ROA.

Fuente. Cálculos propios con base en datos. (Ortíz, 2008). Salidas SPSS.

Tabla 6. Estadísticos ANOVA No.5 (Significancia conjunta)

\begin{tabular}{|l|l|l|l|l|l|l|}
\hline & Modelo & $\begin{array}{c}\text { Suma de } \\
\text { cuadrados }\end{array}$ & GI & $\begin{array}{c}\text { Media } \\
\text { cuadrática }\end{array}$ & F & Sig. \\
\hline 1 & Regresión & 1861,197 & 3 & 620,399 & 18,630 &, $002(\mathrm{a})$ \\
\hline & Residual & 199,806 & 6 & 33,301 & & \\
\hline & Total & 2061,003 & 9 & & & \\
\hline
\end{tabular}

a. Variables predictoras: (Constante), Innovación, Información, Estructura

b. Variable dependiente: ROA

Fuente. Cálculos propios con base en datos. (Ortíz, 2008). Salidas SPSS.

Algunas posibilidades y utilidad de contar con un modelo de pronóstico como el propuesto son, entre otras:

$\Rightarrow$ Contar con un marco de base para establecer hipótesis sobre las variables incluidas, y en muestras de empresas más amplias.

$\Rightarrow$ Establecer proyectos de investigación encaminados a validar tales propuestas.

$\Rightarrow$ Contar con un instrumento para pronosticar rápida y descriptivamente los resultados más probables de un esfuerzo en inversión en la modernización de la gestión empresarial.

$\Rightarrow$ Ayudar a desarrollar planes de intervención empresarial más efectivos y eficientes.
El modelo de regresión lineal calibrado resulta útil para las tareas de pronóstico e inferencia al juzgar por los estadísticos principales. Sin embargo, es posible llegar a conclusiones complementarias mediante un ajuste a la especificación del modelo.

Como alternativa se consideró un modelo no lineal en las variables independientes, pero sí en los coeficientes, del tipo reciproco, y como variable dependiente la variación del indicador ROA con respecto a su media (ROAVar), en los siguientes términos:

$$
\operatorname{ROAVar}=\beta_{\mathrm{o}}+\sum_{\mathrm{i}=1}^{\mathrm{n}} \beta_{\mathrm{i}} \frac{1}{\mathrm{x}_{\mathrm{i}}}+\mu
$$

\footnotetext{
${ }^{6}$ El hecho de utilizar la variación del indicador ROA respecto de la media agrega mejor comportamiento a la estimación del valor, y permite una interpretación en términos de diferenciación respecto de la tendencia media del grupo.
} 
Este tipo de modelos recíprocos tiene la característica que en la medida que aumenta la variable (x) indefinidamente, el término $\beta_{\mathrm{i}} \frac{1}{\mathrm{x}_{\mathrm{i}}}$ se acerca a cero y ROAVar se aproxima al valor límite o asintótico $\beta_{0}$. El mejor ajuste del modelo mediante metodología de eliminación de variables hacia atrás incluye las siguientes variables, reciprocas de las originales:

Tabla 7. Coeficientes modelos de regresión recíproco

\begin{tabular}{|c|c|c|c|c|c|}
\hline Modelo & $\begin{array}{l}\text { Coefici } \\
\text { estand }\end{array}$ & $\begin{array}{l}\text { entes no } \\
\text { arizados }\end{array}$ & $\begin{array}{c}\text { Coeficientes } \\
\text { estandarizados }\end{array}$ & $\mathbf{T}$ & Sig. \\
\hline & B & Error típ. & Beta & & \\
\hline 1 (Constante) & 30,389 & 7,085 & & 4,289 & ,000 \\
\hline R.Información & $-3402,824$ & 833,478 & $-1,814$ & $-4,083$ &, 000 \\
\hline R.Asociatividad & 557,956 & 172,706 & ,777 & 3,231 & ,003 \\
\hline R.Exportaciones & 252,911 & 79,104 & ,493 & 3,197 & 003 \\
\hline R.Innovación & 252,911 & 310,533 & ,748 & 2,187 & ,036 \\
\hline
\end{tabular}

a. Variable dependiente: ROAVar

b. Regresión de mínimos cuadrados ponderados-Ponderada por CIIU

Fuente. Cálculos propios con base en datos Ortiz, 2008. Salidas SPSS,

Las variables introducidas son las recíprocas de información, asociatividad, exportaciones e innovación y conocimiento, con comportamiento en todos los coeficientes significativos a niveles superiores al 95\% de confianza. El ajuste y bondad del modelo son los siguientes.

Tabla 8. Ajuste y bondad del modelo de regresión recíproco

\begin{tabular}{|c|c|c|c|c|c|c|c|c|c|}
\hline $\mathbf{R}$ & $\begin{array}{c}\mathbf{R} \\
\text { cuadrado }\end{array}$ & & Error típ. & \multicolumn{5}{|c|}{ Estadísticos de cambio } & \multirow{2}{*}{$\begin{array}{l}\text { Durbin- } \\
\text { Watson }\end{array}$} \\
\hline & & & & $\begin{array}{l}\text { Sig. del } \\
\text { cambio } \\
\text { en F }\end{array}$ & $\begin{array}{c}\text { Cambio } \\
\text { en } \mathrm{R} \\
\text { cuadrado }\end{array}$ & $\begin{array}{c}\text { Cambio } \\
\text { en F }\end{array}$ & g11 & $\mathrm{g} 12$ & \\
\hline ,739(a) &, 546 & ,487 & 23,77820 & ,546 & 9,316 & 4 & 31 &, 000 & 2,147 \\
\hline
\end{tabular}

a. Variables predictoras: (Constante), R. Innovación, R. Exportaciones, R. Asociatividad, R. Información.

b. Variable dependiente: ROAVar.

c. Regresión de mínimos cuadrados ponderados - Ponderada por CIIU

Fuente. Cálculos propios con base en datos Ortiz, 2008. Salidas SPSS.

Tabla 9. Estadística ANOVA. Modelo de Regresión recíproca

\begin{tabular}{|c|l|l|l|l|c|}
\hline Modelo & $\begin{array}{c}\text { Suma de } \\
\text { cuadrados }\end{array}$ & \multicolumn{1}{|c|}{ gl } & $\begin{array}{c}\text { Media } \\
\text { cuadrática }\end{array}$ & F & Sig. \\
\hline 1 Regresión & 21693,107 & 5 & 4338,621 & 7,700 &, $000(\mathrm{a})$ \\
\hline Residual & 16904,571 & 30 & 563,486 & & \\
\hline Total & 38597,678 & 35 & & & \\
\hline
\end{tabular}

a. Variables predictoras: (Constante), R. Exportaciones, R. Estrategia, R. Asociatividad, R. Innovación, R. Información

b. Variable dependiente: ROAVar

c. Regresión de mínimos cuadrados ponderados - Ponderada por CIIU

Fuente. Cálculos propios con base en datos Ortiz, 2008. Salidas SPSS. 
El modelo enseña un ajuste con un R cuadrado corregido de 0,487. El valor del estadístico Durbin-Watson cercano a 2 certifica la ausencia de autocorrelación. Así mismo, la tabla ANOVA indica un p-valor del contraste de la $\mathrm{F}$ para la significación conjunta de los parámetros estimados menores a 0,05 , indicando que hay alta significatividad conjunta de los coeficientes del modelo.

\section{Hallazgos}

\section{¿Qué podemos lograr entonces, en materia de infe- rencia teórica, a partir de los resultados obtenidos?}

La especificación de los modelos anteriores no resulta trivial a la luz de la teoría. En primera instancia, se ha establecido que el asunto de la gerencia se desarrolla mediante un proceso de comunicación y decisiones (Mintzberg, et al., 1997, p.192) (Kim \& Mauborgne, 2005. p. 248) que opera bajo incentivos del ámbito intangible y económico, por lo que no debe resultar extraño que el factor gerencial de comunicación, que encierra tanto los componentes de comunicación como de información organizacional este presente tanto en el modelo lineal como en la versión recíproca y cuyos coeficientes mantienen las mayores magnitudes, esto es, el mayor efecto en la rentabilidad de la empresa.

Este factor de comunicación e información incorpora al interior del MMGO cuatro conceptos básicos: procesos de documentación formal de las reuniones de la organización; la relación entre datos, información y conocimiento, dentro del marco de la gestión del conocimiento y de la organización que aprende; el concepto de gestión documental y por último, la administración de contenidos de los sistemas de información digitales (Rodríguez, 2008).

En los procesos de gestión la información es un elemento básico de toda decisión y tiene que tomarse tanto de los procesos que tienen lugar al interior de la empresa como de los mensajes que envían el mercado, el gobierno y otros factores externos. Noobstante, el que el insumoinformación se depure, seleccione y almacene adecuadamente, y que llegue efectivamente a los tomadores de decisiones, exige que la organización cuente con una adecuada estructura que le garantice tales resultados y es aquí donde toma importancia la siguiente variable incorporada en el modelo: la estructura organizacional de la empresa.
El proceso de estructura organizacional ha tomado cada vez más importancia en la literatura administrativa (Hall, 1983. P. 91 y 92) (Pérez R., 2007), por razones que parecen ganar cada vez más vigencia. Esto puede apreciarse desde la metáfora biológica evolutiva, que muchos teóricos aceptan, no sin reservas, como un enfoque adecuado para los análisis de fenómenos socioeconómicos (Nelson, 1982) o específicamente de la firma (Nelson, 1994). Bajo este enfoque podríamos considerar que la estructura organizacional, funciona como los órganos que permiten tomar información externa e interna para que funcionen sus procesos de manera adecuada. Con el tiempo, estas estructuras van evolucionando y adaptándose a nuevas necesidades dictadas por el mercado y otras variables del entorno organizacional. Sólo aquellas que logren establecer estructuras adaptadas a las necesidades de su momento histórico, logran perdurar.

La estructura organizacional es más que la respuesta de la empresa a su entorno, también permite generar los recursos necesarios para invertir el proceso, esto es, para hacer que las visiones empresariales se traduzca en hechos en el mercado, en emprendimiento, lo que involucra necesariamente la concepción de una estrategia, implícita o explícita, a la manera de creación destructiva planteada por Schumpeter (1934). Esto podría explicar porque el factor gerencial de direccionamiento estratégico que resultó ser la variable con mayor capacidad discriminadora (ver tabla 1) de las empresas seleccionadas, no aparece en los modelos de inferencia y pronósticos calibrados. Dentro del MMGO el factor de eEstructura organizacional evalúa en su primer descriptor la adecuación de sí misma al sistema de valores practicados por sus miembros (dependiente directamente de la cultura organizacional) y la manera en que la planeación estratégica guía las actuaciones de los mismos. Es decir, que si bien el MMGO observa por separado la planeación y la estructura de la organización, en efecto, la estructura organizacional le da forma, tanto a la planeación como a los modos en que estos se distribuyen en la organización y de esta hacia el mercado.

El tercer factor incluido en el modelo de regresión lineal es la innovación y conocimiento (que es acondicionado y facilitado por la cultura organizacional). La innovación alcanza su manifestación más clara dentro de los sistemas que admiten ventajas de monopolio en entornos competitivos, lo que exige que la empresa disfrute de conocimientos suficientes sobre el mercado aplicable a las innovaciones con el objeto de explotar sus ventajas a 
escala. En otras palabras, la innovación es la estrategia natural de una empresa expuesta a la competencia y deseosa de permanecer y crecer en el mercado (Senge, 2000. P. 462 a 468).

Así mismo, el conocimiento necesario para desarrollar innovaciones diferenciales es un proceso colectivo, que se incrementa gracias a la comunicación entre los actores de la organización (Nonaka y Takeuchi, 1999). Y este proceso de innovación se genera en toda organización en algún grado, ya que esta es la única vía de cambio y ninguna organización puede permanecer sin cambios y no desaparecer.

Por otra parte, en el modelo reciproco (figura 4) se incorporaron dos factores adicionales, el primero es la asociatividad, que pudiera estar indicando el hecho que las empresas que mantienen una rentabilidad superior, independiente del negocio en que se encuentren, lo hacen gracias a la capacidad de aprovechar las oportunidades del mercado y responder de manera flexible a las mismas, lo que, en limitaciones de capacidad instalada se supera gracias a la asociatividad. Pero este procesos no sólo es rentable para mejorar la rapidez de respuesta frente al mercado, también incrementa el poder de negociación de las organizaciones logrando mejores posiciones estratégicas frente a los proveedores y los canales de distribución, además de ampliar los conocimientos de las organizaciones participantes quienes comparten información y habilidades comunes (Pérez, R., et, al. 2009. P. 440 y 441).

Por último, está el factor de exportaciones, lo que no resulta sorprendente pues este proceso implicaun aprovechamiento de los términos de intercambio, establecimiento de redes de comunicación extensas y comprensión de procesos de estandarización globalmente aceptados, o al menos de los países involucrados. Precisa establecer estructuras de mercado adecuadas a la competencia internacional y quizás algún nivel de asociación horizontal o vertical.

Los coeficientes en el modelo de opción reciproco son más lejanos entre sí que los del modelo lineal, y adicionalmente, el coeficiente de la variable información presenta signo negativo. Estas características pueden observarse gráficamente para derivar así algunas conclusiones especulativas sobre el comportamiento de las variables y su efecto en la rentabilidad de las empresas. Si bien no es posible realizar una figura en más de tres dimensiones que represente el modelo completo, podemos, establecer el comportamiento individual de cada factor, como estrategia para aproximarnos a un comportamiento simultaneo.

Figura 4. Variables del modelo recíproco

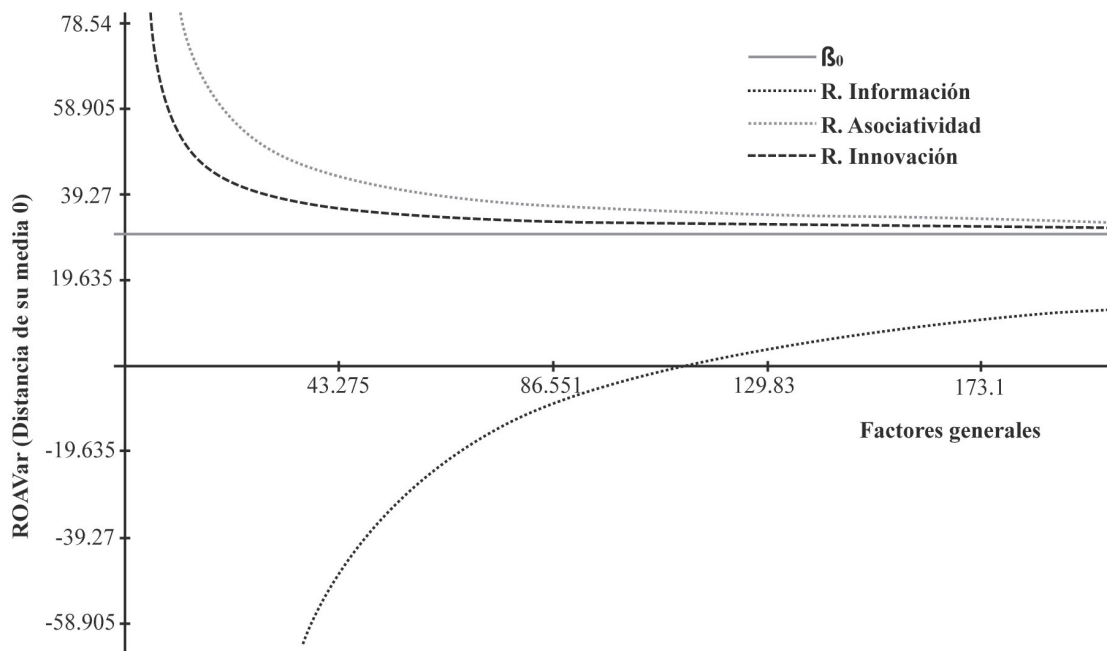

Fuente. Cálculos propios. 
$\beta_{0}$, el valor asintótico, puede interpretarse como el valor que a largo plazo alcanzan las empresas que mantienen los mayores niveles de modernización de la gestión organizacional, con una rentabilidad $30 \%$ superior a la media de conjunto de empresas. Otra forma de interpretar este coeficiente, es como la máxima rentabilidad que la gestión moderna puede agregarle a las Pymes sobre aquellas que se mantienen en niveles de gestión promedio.

La desaceleración en el crecimiento de las trayectorias de las tres variables involucradas señala el proceso de agotamiento en el aporte de los factores gerenciales en la medida que se emplean con mayor intensidad; en tanto que la magnitud absoluta de sus coeficientes indica la velocidad a la que se deteriora dicho aporte. De esta manera, los factores de innovación y asociatividad alcanzan rápidamente sus aportes, límites, en tanto que la información y conocimiento tardan más en asignar sus mayores aportes a la variación de la rentabilidad. Por último, el signo negativo del factor información y conocimiento estaría implicando que este es un proceso inherente a las empresas aún cuando estas estén obteniendo rentabilidades inferiores a la media o incluso negativas.

Los factores gerenciales o componentes organizacionales involucrados en los modelos planteados se pueden contrastar con lo que Pérez R., et al. (2009), señalan como fundamentales en las tareas de modernización gerencial, tanto desde las iniciativas empresariales como de programas de fomento y apoyo gubernamental. Estos factores son reunidos en el llamado "Pentágono de la gestión de excelencia para Pymes" rotulado por Pérez R. (2010) director del grupo de investigación G3PYMES de la Universidad EAN, que son el resultado de extensos y numerosos estudios alrededor de las Pymes colombianas, estos factores son: planeación y estrategia, gestión del mercadeo, cultura organizacional, innovación y conocimiento, y gestión financiera. Con excepción de la gestión financiera, se pueden rastrear íntimas relaciones entre los factores del pentágono de la gestión de excelencia y las variables deducidas como de mayor eficiencia introducida en los modelos de pronóstico e inferencia señalados.

Aún cuando la naturaleza de los datos aquí expuestos no es suficiente para tener observaciones a través del tiempo, podría especularse sobre el lapso en el que las perturbaciones afectan los factores gerenciales, pueden tener consecuencias en la organización, y considerar que los problemas financieros resultan oscilantes, y cuyo control depende dependería de técnicas y recursos estandarizados y expandidos en las prácticas gerenciales que resultan de rápida ejecución, lo que incluye el fácil acceso al crédito.

Para terminar, la utilidad de los modelos aquí diseñados, y en especial de sus posteriores avances y adaptaciones en términos de pronóstico, podrían extenderse a la evaluación de un mayor número de Pymes, quizás de un mismo sector económico, para derivar posibles estrategias de modernización en la gestión con mejores posibilidades de mayor éxito en los resultados financieros de las mismas. Esto podría apoyar especialmente los esfuerzos institucionales de promoción de la Pyme. Igualmente importante podría resultar la oportunidad de contrastar estos resultados frente a la teoría moderna de la gestión y derivar mejoras en las metodologías de diagnóstico e intervención de las Pymes, tal como lo pretende el MMGO.

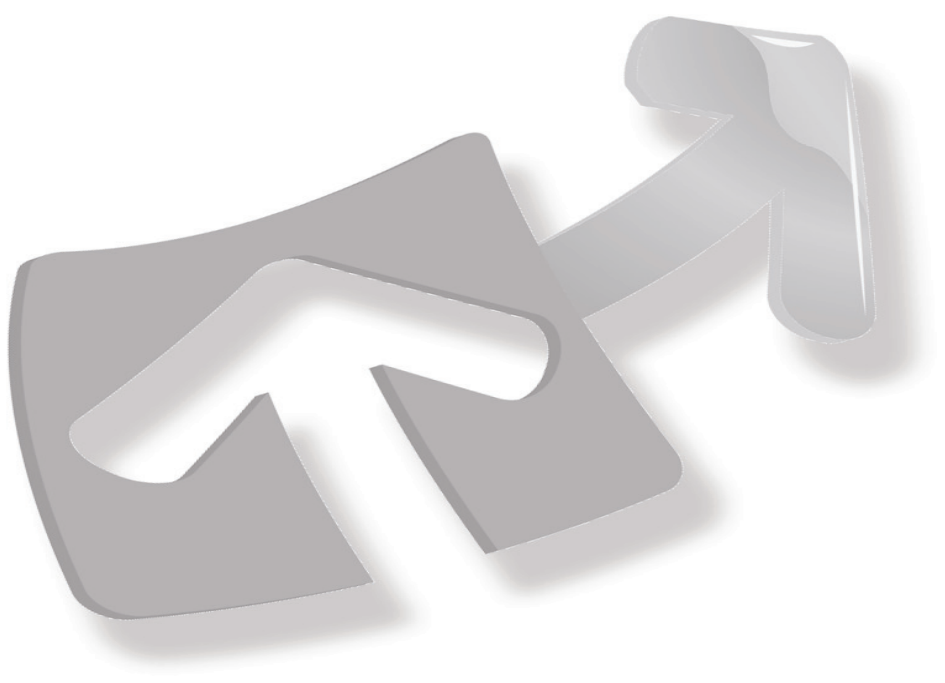




\section{CONCLUSIONES E IMPLICACIONES PARA LA PRÁCTICA Y TEORÍA GERENCIAL Y FUTURAS INVESTIGACIONES}

$\Rightarrow$ La consideración de la gestión organizacional como una función tecnológica que permite combinar factores gerenciales en una determinada forma e intensidad y transformarlos en bienes o servicios valiosos para la sociedad, puede resultar de utilidad para inferir sobre la importancia y el impacto que estos procesos gerenciales tienen en la rentabilidad de las organizaciones.

$\Rightarrow$ Los contrastes entre los modelos de enfoque de la interdependencia y los de pronóstico de la dependencia, parecen indicar que existen factores gerenciales cuyas intensidades de aplicación en las organizaciones, permiten por un lado, discriminar las empresas según sus resultados en rentabilidad y los demás atributos gerenciales que las describen (dimensiones) y, por otro, variables que resultan útiles para pronosticar e inferir resultados posibles en rentabilidad ante cambios en el uso de los factores gerenciales.

$\Rightarrow$ Los factores gerenciales que demostraron tener mayor capacidad para clasificar las empresas según sus atributos son: planeación y estrategia (direccionamiento estratégico), gestión humana, comunicación e información, asociatividad y cultura organizacional.

$\Rightarrow$ En contraste, los factores gerenciales que certificaron ser estadísticamente significativos para pronosticar e inferir teóricamente sobre el impacto de la gestión en la rentabilidad son: información y comunicación, estructura organizacional y conocimiento e innovación.

$\Rightarrow$ El factor de información y comunicación (como componente directamente incluyente y relacionado con el direccionamiento estratégico y la cultura organizacional) destaca como el que más influye sobre los resultados en rentabilidad de la empresa, al juzgar por la magnitud positiva del coeficiente estimado en el modelo econométrico $(1,108)$, le siguen la Estructura Organizacional $(1,092)$ y Conocimiento e Innovación $(0,589)$. $\rightarrow$ El aporte de los factores gerenciales, individualmente considerados, sobre la rentabilidad de las empresas, parece tener un comportamiento no lineal que pierde fuerza en la medida que se incrementa el uso o la inversión en dicho factor. En otras palabras, parece existir un principio de saciedad o agotamiento individual de los mismos.

$\Rightarrow$ Pese al aporte decreciente de los factores en la rentabilidad, los resultados indican que existe un equilibrio a largo plazo para las empresas que invierten en prácticas de gestión más modernas, alrededor de un $30 \%$ superior a la media.

$\Rightarrow$ El hecho que los factores gerenciales, tanto en el modelo lineal como reciproco, presenten coeficientes de magnitudes diferentes, y en el caso del último, signos contrarios, podría estar indicando ciertos tiempos y magnitudes en que estos aportan varianza adicional a la rentabilidad. Esto justificaría posteriores indagaciones sobre la conveniencia de realizar intervenciones o inversiones en tales factores en momentos diferentes de los procesos de modernización de las empresas, y en determinadas magnitudes, con el objeto de alcanzar una mayor efectividad y eficiencia. Es decir, podría tener implicaciones en el diseño de planes de intervención y modernización de las empresas.

$\Rightarrow$ El beneficio adicional generado ante la inclusión de la variable CIIU como ponderador en la estimación de los modelos econométricos, implica que la diversificación sectorial es importante, por lo que se alienta, en la medida que los datos disponibles lo permitan, así como adelantar posteriores estudios empíricos en el ámbito de las actividades económicas que capten estas diferencias. Es probable que beneficios adicionales se logren al realizar segmentaciones de la muestra según criterios de región, antigüedad y de tamaños más específicos.

$\Rightarrow$ Así mismo, se recomienda ampliar el alcance del presente estudio a una muestra más grande de Pymes 
con una visión sectorial, que permita validar los hallazgos aquí expuestos en un marco más amplio y riguroso que permita establecer argumentos de cara a recomendaciones de actuación tanto pública como privada.
$\Rightarrow$ Por último, se insta a establecer un proyecto de trazabilidad de largo alcance que permita verificar los procesos evolutivos de las Pymes que lleguen a ser intervenidas, con recomendaciones derivadas tanto de la aplicación del MMGO como de la naturaleza de los hallazgos aquí mencionados.

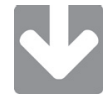

\section{BIBLIOGRAFía}

Arce S. y Cifuentes J. (2006). Gestión del conocimiento: reseña sobre tendencias recientes a nivel mundial. Grupo de Investigación en Management. Vicerrectoría de Investigaciones. Universidad EAN.

Benavides V, C. y Quintana C. (2003). Gestión del conocimiento y calidad total. Madrid: Ediciones Díaz de Santos S.A. y Asociación Española para la Calidad.

Castellanos, G. (2003). Pymes innovadoras. Cambio de estrategias e instrumentos. Revista Escuela de Administración de Negocios No. 47 .Enero-Abril .Págs. 10 - 33

Diaz, L. (2006). Gestión del conocimiento y tecnologías de información y comunicaciones. Revista Escuela de Administración de Negocios No. 58 Septiembre- Diciembre. Pp. 41-59.

Diaz, L. (2002). Informe final del Proyecto "RED-ETI". "Consolidación de la Red Especializada de Centros de Investigación y Desarrollo Tecnológico de Electrónica, Telecomunicaciones e Informática ETI. Proyecto realizado por el Centro de Investigación de las Telecomunicaciones (CINTEL), Instituto Tecnológico de Electrónica y Comunicaciones (ITEC), Centro de Apoyo de la Tecnología Informática (CATI), Centro Internacional de Física (CIF).

Fabrizio, J. et al. (2003). Economics language and assumptions: How theories can become self-fulfilling. IESE Business School - Universidad de Navarra.

Gujarati, D. N. (1997). Econometría. Mc Graw Hill. Tercera Edición,

Hall R. (1983). Organizaciones, estructura y proceso. Tercera edición. Prentice hall.

Hayek, F. A., (1952). The Sensory Order. Chicago: University of Chicago Press.

Kim Ch. y Mauborgne R. (2005). La estrategia del océano azul: Como desarrollar un nuevo mercado donde la competencia no tiene ninguna importancia. Grupo editorial Norma y Harvard Business School.

Minztberg H., et al (1997). El proceso estratégico: conceptos, contextos y casos. Prentice hall. 1997.

Nonaka I. y Takeuchi H. (1999). La organización creadora de conocimiento. Oxford University Press. México. 
Nelson, R. (1982). An Evolutionary Theorty of Economic change. Cambridge: The BelKnap Press of Harvard university.

Nelson, R. (1994). The theory of the firm (II). En: The Elgar companion to institutional and evolutionary economics. Aldershot.

Nieto, M. (2005). Producción y gestión del conocimiento en la actividad académica. Revista Escuela de Administración de Negocios No. 53 Enero-Abril de 2005 P. 36 -51.

Ortiz, W. (2008). Adecuación y actualización del componente financiero del MMGO y desarrollo de una metodología para evaluación del impacto de la gestión en los resultados financieros de las organizaciones. Informe final de investigación. Vicerrectoría de Investigación Universidad EAN.

Pérez, R. (2006). "Liderazgo Visionario: centro del conocimiento". Revista Escuela de Administración de Negocios No. 58. Septiembre a Diciembre de 2006. Págs. $79-85$.

Pérez, R. et al., (2009). Análisis empírico de la aplicación del modelo de modernización de la gestión para organizaciones en Pymes colombianas. Revista EAN, No. 65 Enero-Abril, p. 77-105.

Pérez, R. (2007). Estructura Y Cultura Organización De Las Pymes Colombianas: Análisis En Empresas Bogotanas. Cuadernos de Administración Universidad del Valle No. 38.

Pérez, R. et al. (2009). Modelo de modernización para la gestión de organizaciones. Universidad EAN. Agosto de 2009.

Pérez, R. (2010). El pentágono de la excelencia para las PYMES. Informe de coyuntura PYME. ANIF-Universidad EAN. Edición 32. 2010. ISSN 2011-9755. P. 10 - 19.

Pérez, C. (2005). Métodos estadísticos avanzados con SPSS. Thompson Editores.

Rodríguez, H. (2008). Rutas para la Modernización de Las Pymes. Fascículo No. 4 Universidad EAN - Diario la República.

Schumpeter, J. (1934). La teoría del desarrollo económico. Fondo de Cultura Económica.

Senge, P. et al. (2000). La danza del cambio. Grupo editorial Norma.

Velasquez, A. (2006). Diseño de Organizaciones para la Creación de Conocimiento. Revista EAN No. 58, Septiembre a Diciembre de 2006. Págs. 5 - 25. 